\title{
Regulación convencional, acción sindical y empleados atípicos. El caso del sector de la limpieza de edificios y locales en Valencia
}

\section{Miguel Ángel García Calavia}

Universidad de Valencia. Facultad de Ciencias Sociales. Departamento de Sociología y Antropología Social miguel.a.garcia@uv.es

\section{Resumen}

Distintos estudios evidencian que asalariados atípicos y sindicatos confluyen poco. Continuando el examen de este desencuentro, los convenios colectivos, ¿protegen a los empleados atípicos?, ¿reducen las desigualdades y los integran?, ¿son una plataforma para la acción colectiva?

En este artículo, se va a intentar contestar a estas preguntas mediante un estudio de la regulación convencional en el sector de la limpieza de la provincia de Valencia, en el que las condiciones atípicas son acusadas y están extendidas. El estudio es una de las primeras aproximaciones a la problemática, ya que apenas hay experiencia investigadora en lo que se refiere al primer interrogante y nada con respecto al segundo y al tercero. A pesar de ello, el elevado número de conflictos colectivos e individuales permiten suponer que existen incumplimientos con respecto a las condiciones laborales pactadas. Y si no se cumplen los convenios colectivos, tampoco habrá homogeneización ni integración de los asalariados de la limpieza.

La información procede de fuentes documentales y estadísticas, de los expedientes registrados en el Tribunal de Arbitraje Laboral. También de un grupo de discusión y de ocho entrevistas en profundidad a informantes cualificados.

Palabras clave: negociación colectiva; sindicalismo; acción colectiva; trabajadores.

Abstract. Conventional regulation, union action and atypical employees: The case of the cleaning services industry in Valencia

Several studies show that atypical wage-earners and trade unions rarely converge. In analysing this mismatch, we ask: does collective bargaining protect precarious workers, does it reduce inequality and favour their integration?, and is it a platform for collective action?

This paper aims to answer these questions through the study of a conventional regulation programme in the cleaning service sector of the province of Valencia, in which atypical conditions are already widely extended. This is one of the first approaches to this problem, 
as little research has focused on the first question, and the second and third issues remain unexplored. Indeed, the high number of individual and collective conflicts shows failure to comply with the labour conditions agreed upon. This incompliance with collective agreements will prevent the homogenization and integration of cleaning workers.

The data was drawn from documental and statistical sources (from records in the Local Arbitration Court), as well as from a discussion group and eight in-depth interviews of qualified professionals.

Keywords: collective bargaining; syndicalism; collective action; workers.

\section{Sumario}

El examen de la eficacia de los convenios colectivos en España

Aproximaciones a la protección convencional de los asalariados atípicos en algunos países europeos

La rama de la limpieza de edificios y locales

Perfil social y situación ocupacional de los asalariados de la limpieza

Patronales y sindicatos en el sector de la limpieza

El peso de la regulación convencional en el ámbito sectorial provincial
Representación sindical y unitaria en la limpieza. Protección convencional

Los asalariados de la limpieza: un grupo fragmentado

Regulación convencional, acción colectiva y otras formas de acción en la limpieza

Todavía una eficacia convencional insuficiente, una fragmentación social

Referencias bibliográficas

En este artículo, se examina la eficacia general de los convenios colectivos, especialmente entre los asalariados atípicos, esto es, si sus cláusulas laborales se aplican completamente a todos los trabajadores en sus ámbitos funcionales respectivos. Asimismo, se exploran los tipos de acciones que desencadenan trabajadores y sindicatos en torno a los convenios colectivos. Se centra, pues, la atención en la regulación convencional entendida como una práctica conjunta de empresarios y trabajadores, de sus representantes, ejercida de manera autónoma, para producir reglas que, recogidas en un convenio, han de regir sus relaciones (Reynaud, 2004: 118); una práctica que perdura más allá del establecimiento del convenio en la acción que despliegan las partes para aplicar sus reglas y alterarlas (Jobert, 2002: 21). Los asalariados atípicos son los empleados de forma distinta a lo que hace tres décadas fue la norma dominante: empleo relativamente estable para un único empleador, integrado en un convenio colectivo, ejercido a tiempo completo en un centro de trabajo concreto y que proporciona una amplia protección social (Bouffartigue, 1999: 12).

El examen se lleva a cabo en la rama de la limpieza de edificios y locales de la provincia de Valencia. Esta rama constituye un ámbito adecuado, en primer lugar, porque algunas condiciones atípicas (las contrataciones a tiempo parcial, inestables y la realización de la actividad laboral en centros de trabajo ajenos 
a la empresa que contrata a la mano de obra, es decir, en los inmuebles del cliente) están extendidas y, por tanto, los contingentes de empleados atípicos son cuantiosos. En segundo lugar, porque la rama registra numerosos incumplimientos empresariales de convenios colectivos, como atestigua la elevada demanda de solicitudes de soluciones extrajudiciales de conflictos colectivos, casi cien entre 2001 y 2010, relacionados, mayoritariamente, con diferencias sobre interpretación y aplicación de cláusulas de los mismos. Y, en tercer lugar, la regulación convencional en la limpieza de Valencia se realiza alrededor de un único convenio colectivo, al igual que en otras 32 provincias. Así, puede ser ilustrativa de lo que acontece en el sector, al menos allí donde la provincia es la referencia territorial.

El estudio es una de las primeras aproximaciones a los objetivos planteados en España. En algunas síntesis, se afirma que un buen número de los trabajadores en España, entre otros, los contratados precariamente, los sumergidos, los ocupados en empresas pequeñas y escasamente sindicalizadas, quedan al margen de la regulación vía convenio colectivo, bien porque no existe o bien porque, aunque exista, de hecho no están al amparo de su protección (Miguélez y Rebollo, 1999: 330-331; Escudero, 2004: 28 y 29). En este sentido, para poder hacernos una idea del posible nivel de cumplimiento de los convenios colectivos, es necesario analizar la estructura empresarial de la rama, su mano de obra y su sindicalismo. En todo caso, hay evidencias de incumplimientos de las condiciones laborales pactadas, como muestran los distintos procedimientos de solución extrajudicial abiertos en este sentido.

Los resultados de la investigación de los objetivos apuntados en el parágrafo inicial constituyen los apartados centrales de este artículo. Previamente, se sintetiza literatura existente relacionada con los objetivos examinados. También se exponen los rasgos característicos del sector (actividad, empresas, mano de obra) y de los actores colectivos que protagonizan las relaciones laborales, para comprender mejor el desarrollo de la negociación colectiva.

La información en que se basa el estudio procede de fuentes secundarias. En primer lugar, de los anuarios del Ministerio de Trabajo y de las encuestas de servicios del Instituto Nacional de Estadística (INE). En segundo lugar, de estudios sobre el futuro de la rama efectuados por consultoras. Y, en tercer lugar, de los 4.336 expedientes registrados en el Tribunal de Arbitraje Laboral (TAL) del País Valenciano entre 1997 y 2010, donde se solicitan soluciones extrajudiciales a otros tantos conflictos colectivos y de los que se dispone de información sistematizada por técnicos de la institución. Los de la limpieza se presentan agregados con los de mantenimiento y jardinería, actividades complementarias: 93 expedientes, entre 2001 y 2010. De la lectura de la pretensión literal de las solicitudes, se deriva que tres de cada cuatro son de la rama de la limpieza, aunque hay que precisar que no siempre se puede establecer a cuál de las tres actividades se refiere el expediente. Esto supone reconocer la existencia de una pequeña distorsión en aquellos aspectos analizados con los datos del TAL. La información para el examen procede también de fuentes primarias: de un grupo de discusión con trabajadoras de la limpieza de hospitales y centros 
universitarios y de ocho entrevistas en profundidad a siete sindicalistas (cuatro de la UGT y tres de CC. OO.), como también a un responsable de la patronal.

Con este examen, en buena medida, de carácter exploratorio, se espera contribuir al conocimiento del desarrollo de la regulación convencional, sobre todo, entre los empleados atípicos. Asimismo, al conocimiento de la acción que promueven este tipo de empleados junto al resto y sus representantes alrededor de dicha regulación. Ambas temáticas han sido poco investigadas en el ámbito de la sociología de las relaciones laborales.

\section{El examen de la eficacia de los convenios colectivos en España}

Según el anuario del Ministerio de Trabajo, se firman o se revisan 5.987 convenios colectivos en 2008, que cubren a 11.968.148 asalariados de 1.605.195 empresas en España, esto es, al 71\% de los asalariados EPA de dicho año, debido a la eficacia general de los convenios colectivos (uno de los principios básicos que caracteriza al modelo español de negociación colectiva), esto es, a la extensión automática de las cláusulas pactadas al conjunto de empresas y trabajadores comprendidos en el ámbito de aplicación del convenio. La fragmentación de la negociación colectiva es, pues, elevada. Esto es debido a la forma de estructurarse. En el ámbito funcional, existen dos tipos de convenios: el de empresa y el de ámbito superior a la empresa (básicamente, sectores de actividad). Ahora bien, no todos los convenios sectoriales tienen el mismo alcance territorial: en 2008, el $77 \%$ son de ámbito provincial, aunque existen de ámbito nacional (un 6,8\%), autonómico (un 7,1\%) y otros (un 9,1\%).

La cobertura formal de la negociación colectiva es incompleta, porque existe un número importante de asalariados (un 29\%) que están excluidos de ella. Esto es especialmente preocupante en relación con aquellos que se encuentran en situación de mayor debilidad y bajo mínimos legales. Además, los correspondientes convenios sectoriales no siempre se aplican completamente en las empresas, sobre todo, en las pequeñas (Alós et al., 1989a, 1989b, 1990; Escudero, 2004: 28-29), de modo que tiene lugar una relativización y un vaciamiento de lo acordado, al mismo tiempo que adquiere relevancia otra vía de fijación de las condiciones laborales: la simple imposición empresarial (Escudero, 2004: 29). Por último, se observa que el centro de gravedad de los contenidos de la negociación colectiva se ha desplazado desde la corrección de la precariedad laboral que padecen determinados segmentos hacia la búsqueda de flexibilidad laboral y el aumento de los poderes empresariales (Miguélez y Rebollo, 1999: 330; Escudero, 2004: 31). De ahí que se considere la negociación colectiva como un proceso insuficientemente eficaz, especialmente en lo que se refiere a los empleados atípicos (Miguélez y Rebollo, 1999: 330; Martínez Lucio, 2002: 568-569; Escudero, 2004: 29).

La eficacia de los convenios colectivos, al igual que sus contenidos formales, se relaciona sobre todo con la fuerza de los sindicatos, que varía de una rama a otra y, dentro de cada una de ellas, de una provincia a otra, aunque también con el nivel de organización de la patronal (Miguélez y Rebollo, 1999: 332; 
Martínez Lucio, 2002: 568-569). En este mismo sentido, se asocian con la articulación de la representación de los trabajadores en la empresa, fundamental para la supervisión de la aplicación del convenio en la misma. Legalmente, sólo es obligatoria en las que tienen más de diez trabajadores y en las que tienen entre seis y diez, si así lo decide la mayoría. Por tanto, en la mayoría de las empresas (más del 75\% tienen menos de seis trabajadores y más del $85 \%$, menos de diez), las medidas de representación son inoperantes (Martínez Lucio, 2002: 569; Pérez Infante, 2003: 58 y 92) y no hay control concreto del uso del convenio.

Resulta, pues, obvio que el estudio de la eficacia de la negociación colectiva se ha de efectuar en relación con la presencia sindical y con el tipo y el tamaño de empresa. En este sentido, se supone, en primer lugar, que la negociación colectiva sectorial no constituye un sistema de regulación totalmente efectivo, especialmente en ramas en las que la organización sindical y la representación de los trabajadores son débiles. En otras palabras, la negociación colectiva sectorial es efectiva en las empresas donde hay presencia sindical que controle la aplicación del convenio y que negocie y concrete las materias cuando es menester, lo que no sucede en las que no hay representación, sobre todo en las pequeñas (Miguélez, 2004: 49). En segundo lugar, que la negociación colectiva no es capaz de remediar la precariedad derivada de la reorganización productiva y empresarial emprendida en la década de 1980. Más aún, la función de mejorar la situación y la posición debilitada de los trabajadores pierde peso frente a la de regular de manera flexible las condiciones laborales. A este respecto, se mantiene la diferenciación interna, cuando no aumenta.

\section{Aproximaciones a la protección convencional de los asalariados atípicos en algunos países europeos}

En algunos países europeos, la protección de la contratación colectiva también es insuficiente, sobre todo entre los empleados atípicos, e, incluso, inexistente. Es lo que se deriva de algunas investigaciones en el sector de la limpieza en Gran Bretaña y Francia. En el caso británico, no hay convenio colectivo sectorial, de modo que las condiciones laborales varían según las empresas y los centros de trabajo. Se apunta que las jornadas partidas, los contratos precarios y la intensificación de la carga laboral constituyen dispositivos habituales para conseguir beneficios en las empresas subcontratistas en un sector muy competitivo. Durante mucho tiempo, la mano de obra se muestra refractaria a toda actitud reivindicativa frente a sus condiciones de empleo y trabajo. Ello es debido a la «mordaza» de la precariedad (Scandella, 2009: 122).

En el caso francés, existe un convenio colectivo sectorial, si bien hay un alejamiento frecuente entre lo que se regula a este nivel y lo que se aplica en los centros de trabajo, de modo que, cuanto más pequeña es la empresa, mayor es la posibilidad de incumplimiento de cláusulas del mismo y de que los asalariados se encuentren en situación de precariedad. En este sentido, se apunta que la dinámica convencional tropieza con la impotencia de los sindicatos para hacer 
respetar las normas en los centros, excepto en los grandes, con la correspondiente degradación de los estatutos de empleo y de las condiciones laborales, así como, también, con la incapacidad de los empresarios y de la patronal para regular la intensa competencia entre las empresas del sector (Denis, 2008: 49). Así, la negociación de convenios colectivos sólo desempeña parcialmente su papel de protector de los asalariados de la limpieza. Consecuentemente, las condiciones de empleo son desiguales y polarizadas, y no consiguen, por tanto, la integración profesional de los trabajadores del sector.

La inexistencia e ineficacia de la contratación colectiva en el sector de limpieza se relaciona, en ambos países, con la dispersión de la actividad productiva en los centros de las empresas clientes y el correspondiente aislamiento de los trabajadores que obstaculizan la constitución de colectivos de los que puedan emerger reivindicaciones colectivas. Se señala que la acción colectiva tiende a quedar constreñida a los grandes centros de trabajo y a no rebasar sus límites (Denis, 2008: 54). También se relaciona con las características de la mano de obra, en general, poco cualificada; una buena parte, emigrante y no siempre con los permisos en regla, de modo que los trabajadores temen tanto al paro como a los servicios de inmigración (Scandella, 2009: 122; Denis, 2008: 55). $Y$, en el caso francés, se relaciona con la debilidad sindical y de la representación colectiva, que no asegura la aplicación del convenio en todas las empresas (Denis, 2008: 48).

Teniendo en cuenta estos entornos, se sigue manteniendo que los convenios colectivos no cubren a todos los asalariados, especialmente a los atípicos, y que existe una acusada fragmentación de la mano de obra en la limpieza. Asimismo, que las respuestas colectivas que puedan generarse alrededor de dichos convenios se localizan preferentemente en los grandes centros de trabajo.

\section{La rama de la limpieza de edificios y locales}

La rama de la limpieza tiene una larga trayectoria histórica. Su origen se sitúa en la segunda mitad del siglo XIX en relación con la logística de puertos y ferrocarriles. Ahora bien, su expansión y consolidación como importante actividad económica tiene lugar a partir de finales de los años setenta, con las jubilaciones masivas del personal de limpieza en el sector público y con la externalización, que no ha dejado de ampliarse desde entonces. En este sentido, la rama de la limpieza es un producto ejemplar de esta línea de restructuración emprendida por las empresas en las últimas décadas del siglo XX, puesto que busca minimizar costes, reducir estructuras internas y transferir responsabilidades.

Dos indicadores lo constatan. Primero, la facturación agregada de las empresas de la rama a nivel de España, que registra un comportamiento positivo en el periodo 1995-2008, en que aumenta considerablemente con una tasa aproximada de crecimiento medio anual de un $10 \%$, si bien la cifra de negocio tiende a desacelerarse en 2008, cuando alcanza los 8.396 millones de euros, con un incremento del 6\%, mientras que, en el año 2009, muestra un descenso del 6,5\% (DBK Consultoría, 2011: 23). Segundo, el número de empresas, que 
pasa de 12.402 en 1995 a 17.058 en 2004 y a 25.231 en 2009 (INE, 2009), sin dejar de crecer ningún año, incluso en tiempo de crisis: en 2008, el número era 24.986 (INE, 2008a) y en 2010, 25.700 (INE, 2010).

Esta vitalidad de la rama se apoya en la débil inversión y en la sencilla organización requerida para la creación de una empresa de limpieza, y se materializa en el número importante de empresarios individuales y de pequeñas empresas existentes: en 2009, el 39\% no tiene ningún asalariado y el 44\%, son pequeñas, con plantillas entre uno y nueve trabajadores. En el otro extremo, las empresas de más de 500 asalariados no llegan ni al 0,5\% del total (INE, 2009). La rama de la limpieza presenta, pues, una estructura empresarial polarizada que se encuentra, asimismo, en las cifras de negocio: en el año 2000, el 65\% de las empresas de cuatro trabajadores o menos controlan el $6 \%$ de las cifras de negocio, mientras que el 3\% de las que tienen 99 o más llegan al 63\% (Encuesta anual de servicios 2000, del INE). Se trata de empresas que ofrecen servicios de limpieza para distintos tipos de inmuebles. Algunas de las más importantes son EULEN, CLECE e ISS European Clearing System (GELIM).

La gran mayoría de las empresas poseen un único local o domicilio. Es el caso de casi todas las pequeñas y de buena parte de las medianas. También hay un pequeño número de pymes con proyección de futuro que cuentan con dos o tres delegaciones. Ahora bien, hay grandes empresas que cuentan con más de sesenta delegaciones, lo que resulta significativo de su alcance y, sobre todo, de la dispersión de la plantilla, todavía mayor si se tiene en cuenta que la de cada delegación se distribuye por centros en los que limpian individualmente o en grupo.

La actividad se articula en la rama en torno a relaciones triangulares entre los clientes (empresas subcontratantes), las empresas prestatarias (o subcontratistas) y sus asalariados. Así, la subcontratación constituye uno de los fundamentos del funcionamiento de la rama y determina su desarrollo económico y social en función de los contratos firmados. Estos se establecen a partir de los pliegos de condiciones, compuestos por una parte técnica y una parte financiera. La selección del prestatario por el cliente, en el entorno de licitaciones regulares, sucede a partir de la toma en consideración de estos dos criterios, aunque el del precio resulta determinante.

En la contratación entre clientes y empresas de limpieza, se perciben distintas tendencias, según el entrevistado de la patronal. Primera, un cambio en los contenidos de los contratos en los que ahora se pone énfasis en los resultados y no tanto en los medios, sobre todo en el sector público, donde se pretenden los mismos servicios aunque se reduzca el personal. Segunda, el hundimiento de los precios. Cada contrato firmado sale de un concurso a la baja al que concurren grandes empresas, mayoritariamente españolas; la demanda procede tanto del cliente privado como del público ${ }^{1}$. Tercera, incipiente, la convocatoria de

1. Los edificios y centros donde las empresas del subsector desarrollan su actividad son: oficinas, centros comerciales, centros sanitarios y residenciales, centros educativos, alojamientos turísticos diversos, naves industriales, comunidades y viviendas. 
concursos en los que los clientes no sólo contratan la limpieza, sino también otros servicios auxiliares (mantenimiento, seguridad y jardinería), puesto que buscan que los prestatarios hagan ofertas integradas, de multiservicios. Lo social cuenta, pues, poco en los contratos de un subsector en el que la masa salarial comporta el $80 \%$ de media del precio de coste de la prestación efectuada.

Además, el fenómeno de la subcontratación, con su correspondiente sucesión de contratas, deja su impronta también en la relación con los asalariados, cuya continuidad en sus puestos de trabajo, una vez finalizada cada una de aquellas, constituye el primer objetivo sindical de la regulación colectiva, esto es, la subrogación de la plantilla con todos los derechos y las obligaciones en caso de cambio de prestatario o subcontratista. La articulación de la limpieza en base a contratas de servicios "cerradas» en concursos públicos constituye un riesgo para la estabilidad del empleo de los asalariados, dado que se encuentra directamente afectada por la dinámica de sustitución del adjudicatario de la contrata. De ahí que se demande sindicalmente y se convenga, como regla general, la subrogación del personal. Así pues, estructura empresarial polarizada, subcontratación y competencia fuerte son rasgos característicos del funcionamiento de la rama que se han de dejar sentir en la capacidad de movilización de los trabajadores de la limpieza.

\section{Perfil social y situación ocupacional de los asalariados de la limpieza}

En 2008 (30/9), la rama de la limpieza cuenta en España con 504.105 ocupados, de los que 482.924 son asalariados y 21.181 , ayudas no remuneradas —básicamente, familiares- (INE, 2008b). Esto supone que hay 236.488 ocupados más que en 1998, casi un $90 \%$ más de mano de obra contratada o de ayuda en diez años. En estas cifras no están incluidas las personas que limpian inmuebles en la economía sumergida. De la provincia de Valencia, no hay datos sobre el año 2008, pero en 2009 el número de asalariados es 19.306, según la patronal provincial.

Casi el $80 \%$ de los asalariados de la limpieza son mujeres, un porcentaje que supera al de Francia, Holanda y Bélgica, por tanto, las mujeres están sobrerrepresentadas en la rama. La Encuesta anual de servicios (INE, 2008) no contiene información sobre la edad del personal ocupado. Los únicos datos son los de la Encuesta de servicios industriales de limpieza, del año 1995, en los que se aprecia un colectivo relativamente envejecido, ya que la mitad de los empleados tiene entre 25 y 40 años y un $38 \%$, más de 40 .

En lo que se refiere al nivel educativo, aunque no se dispone de datos, los informantes cualificados apuntan la existencia de gente sin estudios constatable en el tipo de asistencias que han de prestar en las federaciones a quienes acuden a las permanencias sindicales solicitando ayuda y asesoramiento a la hora de rellenar formularios o solicitudes. Igualmente, también se observa la presencia de inmigrantes, algunos de ellos, en situación irregular. Su número en la limpieza es difícil de fijar. 
Una última característica del perfil social de las limpiadoras es que algunas de ellas, probablemente más que en otros colectivos de trabajadores, se encuentran en situaciones familiares difíciles: encabezan hogares monoparentales o forman parte de familias numerosas.

En lo que se refiere a la situación ocupacional, un $40 \%$ de los empleados en la rama en el año 2008 están contratados como eventuales y el 60\% restante, como contratados indefinidos (INE, 2008b). La Encuesta anual de servicios (INE, 2008b) tampoco informa sobre si los ocupados lo son a tiempo completo o a tiempo parcial. En la Encuesta de servicios industriales de limpieza, de 1995, se recoge que casi la mitad de los empleados lo son a tiempo completo (un $48 \%$ ), mientras que el $52 \%$ lo son a tiempo parcial. En este caso, los varones se encuentran proporcionalmente bastante más entre los primeros (un 73\%) que las mujeres (un 40\%). Ahora bien, han pasado bastantes años. Los informantes cualificados reconocen la importancia de la ocupación a tiempo parcial, aunque no precisan cifras. De este modo, las condiciones de empleo se polarizan, no solo entre indefinidos y temporales, sino también entre contratados a tiempo completo, localizados predominantemente en grandes centros de trabajo y en grandes empresas, y contratados a tiempo parcial, localizados en pequeñas y medianas empresas.

\section{Patronales y sindicatos en el sector de la limpieza}

La polarización en el tejido empresarial de la limpieza se refleja en la representación colectiva de los intereses correspondientes. Por un lado, se encuentra la Federación AFELIN, que reúne a 18 asociaciones de 28 provincias españolas con más de mil empresas de limpieza que emplean a 190.000 trabajadores, según la web de la propia federación. El grado de asociacionismo empresarial es bajo, teniendo en cuenta el número de empresas del sector. AFELIN representa a pequeñas y, sobre todo, a medianas empresas. Esta federación apenas negocia, ya que no hay convenio estatal. La negociación de convenios de ámbito provincial y autonómico está protagonizada por las asociaciones provinciales o autonómicas respectivas. Por otro lado, se encuentra la Asociación Profesional de Empresas de Limpieza (ASPEL), asociada a la CEOE. Representa a las grandes empresas del sector (16) y está siempre presente en la negociación colectiva, cualquiera que sea su ámbito. También en la provincia de Valencia, junto a la Asociación Provincial de Empresarios de Limpieza (APELVA), a la que pertenecen 34 empresas.

Aunque no hay datos precisos facilitados por las federaciones sindicales correspondientes, se apunta que la afiliación a los sindicatos es baja (o muy baja). Según el estudio del sector, elaborado por ACITE (2002), la presencia de afiliados a la UGT se localiza en un 6\% de las empresas y es ligeramente superior a la de CC. OO. La afiliación sindical se encuentra especialmente en las empresas de más de 500 trabajadores y es inexistente en las de menos de 20 trabajadores (ACITE, 2002: 40). Existe, pues, una relación directa entre tamaño de la empresa y afiliación sindical. Unos porcentajes que matizan los 
informantes cualificados, al menos a nivel de la provincia de Valencia, así como la distribución de la afiliación, que, como se expondrá más adelante, se localizan tanto en las grandes como en las pequeñas empresas.

El volumen electoral de ambos sindicatos es mayor, si bien tampoco se dispone de cifras exactas sobre la participación de los trabajadores. En febrero de 2006, los resultados de las elecciones a órganos de representación en las empresas son que de 9.871 delegados elegidos en España, de los cuales, 4.454, es decir, el $45 \%$, corresponden a CC. OO., y 3.669, es decir, el 37\%, a la UGT (Baylos y Trillo, 2006: 27). En determinadas provincias, se reproducen estos resultados y, en otras, es UGT la que gana seguida de CC. OO. En el caso de la provincia de Valencia, es UGT la que obtiene la mayoría en las elecciones de 2006 y 2008.

Las agrupaciones sindicales respectivas de los trabajadores de la limpieza, tanto de CC. OO. como de UGT, no constituyen organizaciones autónomas dentro de ambos sindicatos, sino que se encuentran integradas en sendas federaciones: en la de actividades diversas (jardinería, seguridad privada, saneamiento urbano), en el primer caso, y en la de servicios (banca, artes gráficas, seguros, oficinas), en el segundo caso.

Las principales reivindicaciones de ambos sindicatos en los últimos diez años han sido: el aumento de los salarios base, que son muy bajos (desde el año 2009 unos 850 euros mensuales); el traspaso a los mismos de los pluses de transporte, con el fin de mejorar la base de cotización; el mantenimiento del plus de antigüedad; el establecimiento de un complemento que asegure el salario íntegro en caso de baja laboral; la reducción de las discriminaciones por género; el mantenimiento de los días de libranza, y la adopción de algún tipo de promoción interna que estimule a la mano de obra. Las reivindicaciones empresariales son: la moderación salarial; la eliminación de los pluses, especialmente el de antigüedad; el aminoramiento de los días de libranza; la reducción del absentismo, y la mejora de la productividad.

\section{El peso de la regulación convencional en el ámbito sectorial provincial}

La rama registra una profusa actividad convencional mayoritariamente de ámbito provincial, cuyos antecedentes básicos se encuentran en el entorno que configura la Ordenanza de la Limpieza aprobada en marzo de 1975 y que mantiene su influencia hasta mayo de 2005, en que las asociaciones ASPEL y AFELIN, por un lado, y UGT y CC. OO., por otro, aprueban un acuerdo marco para la limpieza, por tanto, más allá de 1994, en que se deroga el sistema franquista de ordenanzas mediante una reforma legal. Dicha ordenanza nacional encuadra la regulación de la actividad y puede ser completada y mejorada por los convenios provinciales. Esta distribución de ámbitos normativos no se modifica ni con la aprobación del estatuto de los trabajadores en 1980, ni tan siquiera inmediatamente después de la derogación del sistema de ordenanzas, ya que se prorroga durante once años más.

El Acuerdo Marco de Limpieza (AML) regula gradualmente una serie de materias. En un primer bloque, la subrogación, una institución central en el 
modelo de relaciones de empleo vigente en el sector, y el régimen disciplinario. La subrogación de personal ante la sucesión de contratas se refiere a un modo atípico de adscripción de los trabajadores a las empresas, según el cual, al término de la concesión de una contrata, pasan a estar adscritos a la nueva titular de la misma. Para que se produzca la subrogación, se deben cumplir algunos requisitos que guardan relación con la antigüedad en la contrata (cuatro meses) antes de la finalización del servicio. En un segundo bloque, se prevé la regulación en un tiempo de las categorías profesionales, la promoción y la estructura salarial. Este Acuerdo Marco de Limpieza se presenta como un texto normativo y de eficacia general, de modo que se ha de aplicar en todo el territorio español.

En este entorno, donde se aprecia el peso de la historia, sindicatos y patronales desarrollan la negociación colectiva focalizada en el ámbito provincial, aunque, en algunas comunidades autónomas (Aragón, Cataluña, País Vasco), se promueven convenios autonómicos y, en siete provincias, se desdobla la contratación colectiva entre limpieza de edificios y locales, por un lado, y limpieza de centros sanitarios (en cinco) y aviones (en dos), por otro.

En el caso de la provincia de Valencia, la acción negociadora, especialmente de los sindicatos, se concentra también en este ámbito, aunque desde hace unos años se piensa en la posibilidad del autonómico para evitar las desigualdades de una provincia a otra. Asimismo, se opta por un único convenio para todo el sector, de modo que no se acepta su desdoblamiento en edificios y locales y centros sanitarios como en Alicante. La razón sindical es preservar la unidad y reforzar la posición de los empleados más débiles con la de los más fuertes y mejorar su situación laboral.

Esta concentración de la actividad convencional en el ámbito de rama profesional a escala provincial guarda relación con los dispositivos institucionales generados históricamente, así como con la estructura y el funcionamiento del propio mercado laboral: la mayor parte de la mano de obra está poco cualificada y su rotación es elevada. También, se corresponde con el peso importante de las medianas empresas. Estos elementos favorecen una actividad convencional sostenida en la que se han aprobado cláusulas en los sucesivos convenios colectivos que equiparan salarialmente a los peones especialistas (muy pocos y varones) y a los limpiadores (muchos y, abrumadoramente, mujeres), un logro del que se sienten especialmente satisfechos los sindicatos, porque corrige una larga discriminación de género; también recogen incrementos salariales porcentuales por encima del IPC, el trasvase del plus de transporte al salario base o la percepción del complemento que asegura el salario íntegro en caso de incapacidad transitoria. Estas dos últimas conquistas son importantes para un colectivo en el que hay bastantes mujeres mayores con pocos años cotizados, así como con determinadas dolencias no graves, como osteoporosis, pero cuya mejora requiere estar de baja.

Se observa, pues, la importancia que tienen las demandas relacionadas con el salario en el sector, nada extraño además teniendo en cuenta que son bajos: mantenimiento de primas (de antigüedad), revisión de salarios, su equiparación cuando se efectúa el mismo trabajo, etc. También hay quejas sindicales contra 
la intensificación del trabajo y las malas condiciones para su realización, aunque no se traducen en reivindicaciones sectoriales.

El último aumento significativo, un 4\%, se consigue en el año 2005. El convenio que recoge el aumento se firma tras una amplia movilización que incluye una huelga indefinida que hace visible la importancia de la limpieza en los centros sanitarios y educativos de la provincia de Valencia. El mantenimiento de la convocatoria de huelga por parte de CC. OO. ante el acuerdo entre UGT y patronales o su desconvocatoria y la firma de un acuerdo salarial por debajo de la demanda inicial, un 7\%, desencadena la ruptura de la unidad de acción entre ambos sindicatos que aún no se ha restablecido. Hasta entonces, elaboran y defienden conjuntamente una plataforma reivindicativa. Asimismo, forman parte de la comisión paritaria, pero, a partir de la ruptura, CC. OO. queda fuera. De este modo, el pobre paritarismo existente es mutilado: queda reducido a patronales y UGT.

A pesar de la ruptura sindical, esta actividad convencional dota a los asalariados de un mínimo estatuto laboral que, además de incluir las mejoras apuntadas, protege a los asalariados de la inseguridad y de los efectos negativos de una fuerte rotación en la rama mediante cláusulas específicas, como las que garantizan el empleo y la continuidad del contrato laboral en caso de cambio de prestatario (la subrogación) y las primas de antigüedad.

\section{Representación sindical y unitaria en la limpieza. Protección convencional}

La limpieza es una actividad de subcontratación que se efectúa en los distintos inmuebles del cliente, por tanto, los centros de trabajo en los que se pueden localizar las limpiadoras son muchos. Su presencia es frecuentemente corta y fuera del horario en que la empresa subcontratante desarrolla su actividad:

La plantilla de limpieza de los colegios públicos de Valencia está constituida por 242 trabajadores diseminados en 90 centros. Por tanto, en cada centro, hay dos o tres trabajadores. Llegar al trabajador es muy costoso. La gente tiene horarios diferentes. Sabemos que se puede empezar a las siete y que se puede acabar a las once de la noche, pero unos empiezan a una hora y otros, a otra. $(\text { RASCO })^{2}$

Además, las relaciones entre los propios trabajadores son casi inexistentes, «ya que, aunque pertenecen a la misma empresa, trabajan en centros distintos y no se conocen", con los correspondientes efectos diferidos a la hora de

2. Las personas entrevistadas entre mayo y julio de 2010 son: RPA, representante de la patronal valenciana; RASUGT, responsable sindical de la federación de servicios de la UGT del País Valenciano; SSLUGT, responsable de limpieza de la UGT del País Valenciano; SUGT1, sindicalista de FES UGT; SGEUGT, sindicalista de la UGT de la empresa GELIM; RASCO, responsable sindical de la federación sobre actividades diversas de CC. OO.; SICO1, sindicalista de limpieza de CC. OO.; SICO2, sindicalista de limpieza de CC. OO. La composición del grupo de discusión realizado en el mismo periodo de tiempo es: tres trabajadoras de hospitales, dos de centros educativos y dos de universidades. 
emprender una acción colectiva: «¿Cómo voy a reclamar dinero cuando no sé lo que hacen los compañeros?, ¿cómo voy a protestar?», expone y explica el mismo responsable de acción sindical. Un razonamiento que ratifica una inmigrante afiliada a CC. OO. en el grupo de discusión es el siguiente: «Aunque siempre he tenido el apoyo del sindicato, el calor de la gente lo tuve cuando me trasladaron de un colegio a la universidad: entonces sentí que los problemas no los tenía yo sola». El alcance del aislamiento y del desconocimiento llega al extremo de que ni siquiera se conocen todos los miembros del comité en determinadas empresas, amplía otra responsable sindical (SICO1).

En estas circunstancias, los sindicalistas, una buena parte de ellos miembros de las instituciones de representación colectiva en las empresas, buscan la viabilidad de la acción reivindicativa:

Explicando a cada trabajador qué vamos a pedir o qué vamos a hacer, de viva voz, la gente tiene dificultades para leer, poco tiempo; esto no siempre es fácil. Dada la diseminación de centros, se necesitarían incluso más delegados, porque a veces están muy cerca unos de otros, pero otras están muy lejos. (RASCO)

Únicamente, en los grandes centros en los que se concentra mucha gente: «[...] esto es, hospitales, grandes centros oficiales, se efectúan asambleas para informar, algunas concentraciones para protestar» (SICO2).

Se observa, pues, que la forma de estructurar empresarialmente la limpieza condiciona la práctica sindical, que tropieza con dificultades que no existen en las ramas donde la actividad laboral de las empresas es realizada en uno o en pocos centros. También condiciona la constitución de los organismos de representación colectiva. Aunque no se dispone de datos sistematizados a escala provincial, las opiniones de los sindicalistas es que se vota menos que en otros sectores, entre otras razones, porque los procedimientos establecidos no contemplan la arquitectura de la rama. La diseminación de centros, los horarios de trabajo, los mismos contratos parciales, la distancia al lugar de la votación son circunstancias que no facilitan acudir a votar. La misma elaboración de candidaturas no es fácil: «[...] si se presentan a delegadas es por hacerte un favor». Tampoco lo es que los delegados sepan desenvolverse por sí mismos, al menos, al principio: «Son personas que no se preocupan por las cosas que a nosotras nos preocupan. Por tanto, personas con las que partes de cero. Hay que formarlas. Aunque son muy dispuestas y tienen ganas» (RASUGT).

Asimismo, la organización laboral de la limpieza, en la que la figura del encargado es básica en la asignación de centros a los trabajadores y en el control de su trabajo, influye en el desarrollo y en el desenlace de las citadas elecciones, «ya que conocen el número de centros y su ubicación, los horarios de los trabajadores» (información necesaria para organizar la campaña sindical), "pueden facilitar o dificultar la presencia de los activistas de los sindicatos, o de los de unos sindicatos en perjuicio de otros", e incluso influyen en ocasiones a la hora de votar, ya que «la gente no conoce a los candidatos» (SICO1). 
La representación colectiva se despliega, pues, con dificultades, y no en todos los centros y empresas, sino en las grandes y en algunas de las medianas. Así, una buena parte de los asalariados no tiene representación colectiva, por tanto, no dispone de la misma para plantear y negociar sus demandas, además, sufre dificultades para acceder a las actuaciones sindicales, con la correspondiente sustitución de la defensa individual por la defensa institucional:

[...] a partir de [su] existencia, el sindicato puede solicitar información o lo que sea a la empresa. Ya no es el individuo, sino el sindicato quien se relaciona y trata con la empresa $y$, si no responde, se le reclama formalmente [...] Las pequeñas y medianas empresas tienen miedo a la inspección, a la judicialización [de la vida en la empresa], lo que no es el caso de las grandes. (RASCO)

Los mismos hechos explican la distribución de la afiliación sindical, aun cuando la ausencia de información precisa en el sector hace difícil su examen. Según los datos de algunos responsables sindicales que contradicen los datos de la encuesta de ACITE (2002), las tasas provinciales son similares a las medias generales e incluso superiores si se introduce el factor corrector que supone que una parte de los asalariados del sector lo son a tiempo parcial. Existen secciones sindicales en hospitales y en centros de trabajo que concentran personal, aunque hay también afiliados en pymes, sobre todo por razones instrumentales: «[...] los problemas son frecuentes, de manera que la disposición de servicios de asesoramiento y defensa compensa el pago de una cuota alta para los sueldos del sector». De todos los problemas que encuentra la sindicación, «el mayor es la dispersión y el aislamiento». Este último imposibilita la construcción de secciones sindicales. Por otro lado, hay que señalar que la condición de afiliado no proporciona por sí misma capacidad contractual al trabajador. Esta última sólo la proporciona la representación colectiva institucionalizada.

Una de las principales consecuencias de que una buena parte de los trabajadores de la rama no tenga representación colectiva (y de la débil organización sindical y de su distribución) es que los convenios no se aplican en su totalidad en las empresas:

Entre las que no están afiliadas a APELVA, son muchas y muchas las cláusulas que no se respetan. Entre las que están asociadas, en las pymes, se suelen aplicar más, aunque cuando no es una es otra la que incumple el pago de alguna prima, los días de libranza. En las grandes es donde más se acatan, aunque no es infrecuente que hagan una interpretación sesgada de alguna cláusula que afecta a mucha más gente. (RASCO)

Lo reconoce un directivo de APELVA: «[...] hay mucha empresa intrusa en el sector que no ajusta las condiciones laborales a las mejoras sociales del convenio, que, por otro lado, están completamente desfasadas en la actualidad».

Por tanto, cuanto más va la empresa por libre, menos respeta las cláusulas de los convenios; cuanto más reducido es el tamaño de la empresa, mayor es el riesgo de que incumpla las decisiones convencionales, mayor es la posibilidad 
de que los asalariados se encuentren en situación de inseguridad, y mayor es también la probabilidad de que las empresas asociadas padezcan la competencia desleal de las que no lo están. Una conclusión se puede extraer: hay distancia entre lo que se regula a nivel de sector y lo que se aplica en el centro de trabajo, de modo que el problema no es tanto el contenido del convenio, sino su aplicación concreta.

En este entorno, se aprecia la impotencia de los sindicatos para hacer aplicar las cláusulas aprobadas en convenios sectoriales en los centros de trabajo, excepto en los grandes, con la correspondiente degradación de las condiciones laborales. Asimismo, se observa la incapacidad de las patronales para regular la competencia cada vez más «salvaje» entre las empresas del sector. Ambos hechos constatan lo observado en el sector de la limpieza en Francia.

No resulta suficiente la existencia de un convenio para proteger a los asalariados atípicos y para mejorar sus condiciones de manera generalizada.

\section{Los asalariados de la limpieza: un grupo fragmentado}

La vida laboral de los trabajadores de la limpieza se desarrolla en alguno (o algunos) de los inmuebles de los clientes, esto es, en alguno de los centros de trabajo. En este sentido, el centro de trabajo, elemento característico de las relaciones de subcontratación, constituye la realidad laboral de los asalariados de la limpieza. Puede ser de tamaño variable: el de un establecimiento bancario o comercial con uno sólo, pero también el de un hospital con algunos centenares y, entre uno y otro extremo, muchos centros con unos pocos. Dispersos en los centros que pueden estar lejos de la sede de la empresa de limpieza, los asalariados apenas mantienen contactos con ella, si no es por mediación del encargado; los pretextos, la ropa de trabajo, la nómina y poco más. No es extraño, pues, que los trabajadores se sientan más vinculados al centro que a la empresa de limpieza, ya que del primero no se suelen mover, mientras que de empleador pueden cambiar varias veces: «El trabajador se considera limpiador del banco "X", del hospital "tal"» (SICO2).

Este vínculo con los clientes no es, de todos modos, muy fuerte, aun cuando «los trabajadores de limpieza se portan bien con ellos, que si hay que hacer tal cosa, pues, se suele hacer proyectando una buena imagen de una empresa (o de unas empresas) que no los reconocen» (RASUGT). Por un lado, los clientes consideran a menudo a los limpiadores como personal externo que efectúa una tarea poco reconocida y valorada. Por otro, porque los trabajadores no limpian dentro de la jornada habitual de las empresas clientes, por tanto, no coinciden con su personal. Esto supone que las franjas horarias para limpiar sean limitadas y que la demanda laboral sea fuerte. Ello explica la extensión del empleo a tiempo parcial en el sector:

Hay muchos contratos a tiempo parcial, desde media hora hasta dos o tres horas. Esto es debido a las características del sector: hay que limpiar cuando no hay actividad en el negocio del cliente, cuando la oficina o la tienda está 
cerrada, y como esto tiene lugar a la misma hora, pues, en determinadas franjas horarias, se necesita mucha gente. (RASUGT)

Además, la limpieza se efectúa más rápidamente cuando los locales están vacíos de sus ocupantes habituales. Así, la proliferación del empleo a tiempo parcial en la rama, por tanto, del empleo atípico, es debida a las condiciones de explotación impuestas por los clientes a las empresas prestatarias, así como a las condiciones de competencia económica imperantes.

Ahora bien, este importante cuarteamiento del trabajo asalariado en la limpieza no solo está relacionado con la estructuración del espacio y del tiempo laboral en la rama, sino también con los ámbitos configurados de negociación colectiva. En el Estado español, hay 46 convenios colectivos provinciales, 10 convenios de comunidad autónoma uniprovincial y 5 de comunidad autónoma pluriprovincial. En esta densa red de convenios colectivos que estructura la negociación y la regulación del trabajo asalariado en la limpieza, se aprecia un despliegue de algunos convenios de carácter autonómico y muchos provinciales; también, una subdivisión de la rama en unidades con entidad negociadora propia (servicios de limpieza hospitalaria, aeroportuaria o de centros educativos).

En la provincia de Valencia, no hay más que un convenio colectivo provincial, esto es, no hay subdivisión de la rama como en la provincia de Alicante. Aun siendo único, los trabajadores de la limpieza de centros sanitarios disponen de más días de libranza anual que el resto de trabajadores de limpieza de otro centros (trece los primeros y siete los segundos), fruto de su mayor actividad reivindicativa, expresada especialmente en los conflictos de finales de los años ochenta. Los asalariados de la rama no tienen, pues, exactamente los mismos derechos; tampoco las mismas experiencias reivindicativas. Una parte disfruta de más días de libranza arrancados a la salida de un conflicto y mantenidos luego en el curso de la negociación colectiva.

Esto no implica que los sindicalistas se conformen con este estado convencional. Al contrario, intentan corregir las desigualdades y cambiarlas. Para ello, utilizan las mejores cláusulas de los convenios de otros lugares como referencia en sus reivindicaciones dentro de su estrategia negociadora. Tampoco se acomodan algunos grandes empresarios que buscan sortear las cláusulas elaborando una oferta más amplia de servicios, lo que les permite escapar del convenio de limpieza y ajustar las condiciones salariales y laborales a normas convencionales más bajas, al mismo tiempo que se introducen y se posicionan en nuevas franjas de negocio. La rama se convierte, así, en un ámbito de disputa que enfrenta a unos asalariados que dependen del convenio de la limpieza con unos empresarios que buscan introducirse y posicionarse en otros sectores de servicios, si bien ajustando los estatutos salariales a las normas convencionales más bajas.

Los asalariados de la limpieza de la provincia de Valencia están, pues, lejos de constituir un grupo afectado uniformemente por el empleo atípico. Más bien se trata de un grupo fragmentado, con desigualdades internas, relacio- 
nadas con el tipo de empresa que los emplea, el tamaño del centro en el que trabajan, el conjunto de normas laborales de las que se benefician, la existencia o no de una representación colectiva. Una vez más, los asalariados que trabajan de manera aislada en centros muy pequeños por algunas horas son los que experimentan con más intensidad los efectos de la individualización de la relación de empleo, no solamente porque se encuentran solos frente a su empleador y a su trabajo, sino también porque están alejados del ámbito de acción de las organizaciones sindicales.

\section{Regulación convencional, acción colectiva y otras formas de acción en la limpieza}

En la limpieza de edificios y locales de la provincia de Valencia, existe una regulación convencional protagonizada, sobre todo, por la patronal APELVA y por las federaciones correspondientes de los sindicatos mayoritarios: UGT, con una representatividad superior al 50\%, y CC. OO. Las relaciones entre sindicatos no son fluidas y ello no sólo se da entre mayoritarios (UGT y CC. OO.) y minoritarios (CGT, USO y algunos otros), sino incluso entre los primeros: las relaciones entre UGT y CC. OO. están rotas a nivel provincial desde finales de mayo de 2005, cuando la primera negocia y firma una propuesta por debajo de la demanda inicial en medio de una movilización general. Además, una buena parte de las relaciones sociales en los centros de trabajo son débiles y no suficientemente atendidas por los sindicatos.

En este entorno, tienen lugar conflictos laborales, como atestiguan las solicitudes de solución extrajudicial registradas en el sistema autonómico, el Tribunal de Arbitraje Laboral (TAL). La mitad de los 91 tramitados entre 2001 y 2010 están motivados por cuestiones salariales; una sexta parte, por cuestiones de jornada, calendario y vacaciones, y otra parte igual, por los mismos procesos de negociación; por tanto, por cuestiones relacionadas con la regulación convencional.

De estos 91 conflictos, 85 son de ámbito de centro de trabajo. En el País Valenciano, el porcentaje es similar, porque, de los 197 conflictos registrados, 14 son de ámbito sectorial. Más del noventa por ciento afectan a toda la plantilla. En veinte de los conflictos de Valencia, se aprueba una convocatoria de huelga, por lo que han de pasar obligatoriamente por el Tribunal de Arbitraje Laboral y en once de dichos conflictos se encuentra solución en los procesos de mediación. No se efectúa, pues, más que en nueve. Dos de ellas tienen carácter sectorial, una en 2001 y otra en 2005, con motivo de la renovación del convenio colectivo. Estos conflictos laborales traducen la existencia de ciertas formas de acción colectiva en el interior de algunos centros de trabajo, pero hay pocos que rebasen sus paredes y pocas medidas de presión que aúnen unos centros con otros.

En el año 2005, con motivo de la negociación del nuevo convenio colectivo en la provincia de Valencia, hubo una extensa e intensa movilización en la segunda quincena de mayo: manifestación de más de mil personas, dos con- 
centraciones ante la sede de la patronal y huelga indefinida, a la que la Generalitat Valenciana responde con unos servicios mínimos del cien por cien. Los dos sindicatos más representativos demandan, de manera unitaria, entre otras reivindicaciones, un aumento de un $7 \%$ de los salarios dentro de una campaña de apoyo a la negociación del convenio en los centros de trabajo. La Dirección General de Trabajo de la Generalitat Valenciana media, tras unos días de movilización, para que las partes lleguen a un acuerdo. Las negociaciones continúan y se alcanza un principio de acuerdo entre APELVA y ASPEL, por un lado, y UGT, por otro, que recoge parcialmente las demandas sindicales: un incremento salarial de un 4\%. CC. OO. mantiene la movilización algunos días más, a fin de intentar conseguir las reivindicaciones completamente. Se rompe la unidad de acción sindical en la provincia de Valencia, que solo se ha rehecho en una ocasión en 2010 y otra más recientemente, cuando se solicitó la renovación del convenio (en Castellón y Alicante, la unión es algo mayor).

Más de la mitad de los conflictos (49) tienen lugar en centros con plantillas superiores a 50 trabajadores; 22, en centros con plantillas entre 11 y 49; 10, en centros con 10 o menos; 10, en ámbitos sectoriales, y de 2 no hay nada escrito. Esto es, la gran mayoría, en centros en los que se reúnen importantes contingentes de limpiadoras. El tamaño de las plantillas de los centros es, pues, una de las variables que explica que la conflictividad y la acción colectiva se localicen más en unos que en otros: en Valencia, en hospitales o en alguna gran empresa como la Ford, y en Alicante, en el aeropuerto del Altet. Las noticias disponibles de prensa (2003-2006) confirman estos datos.

En la provincia de Valencia, las soluciones extrajudiciales de los conflictos colectivos son solicitadas por los propios sindicatos y por las instancias de representación colectiva: en casi la mitad (40), CC. OO. o representantes o delegados pertenecientes a este sindicato; en 21, UGT o representantes del sindicato; en 12, CGT, y en 6, USO. En el País Valenciano, el recurso a los procedimientos del TAL es semejante. En este sentido, los conflictos tienen lugar en centros que no solo son grandes (o medianos), sino que cuentan además con presencia sindical institucionalizada, lo que no se produce en los pequeños. Así, el mapa de la conflictividad en la rama de la limpieza guarda relación con el de la sindicalización: existe donde la organización y la actividad sindical es importante y no existe donde no hay.

Además, hay que señalar que el estatuto atípico de empleo, esto es, el empleo a tiempo parcial e inestable, que tiene casi la mitad de la mano de obra del sector, no favorece la acción colectiva. La precariedad laboral, al igual que los bajos niveles medios salariales, convierten la huelga en una acción demasiado penalizada y arriesgada. Por otro lado, resulta complicada de organizar desde un punto de vista práctico, cuando los trabajadores están a tiempo parcial y con varios empleadores.

Las dificultades en la acción sindical y colectiva explican, en buena medida, la importancia que adquieren las consultas y los recursos individuales en la rama de la limpieza: «[...] hay muchas consultas, guardan relación mayoritariamente con incumplimientos del convenio colectivo». Sobre todo, en referencia 
al salario: «Mi experiencia me lleva a decir a las trabajadoras: "Si me traes la nómina, con las diferencias que encuentre, te pagas la cuota de afiliación del año" ", pero, también, con los días de libranza, de permiso retribuido, con los derechos que generan las modalidades contractuales en el sector o con la subrogación cuando se produce, ya que:

A la gente le angustia mucho no saber qué empresa vendrá, qué encargado tendrá. Por otro lado, ¿̇cómo voy a denunciar al Ayuntamiento? El alcalde me ha hecho el favor de colocarme y me ha prometido que tendré el trabajo para largo. (RASUGT)

La magnitud de las consultas (y de los recursos) es tal que, al menos, UGT y CC. OO. organizan permanencias jurídicas para atender a los asalariados y ayudarles a resolver sus problemas y preocupaciones:

Las consultas son muchas; tantas, que las permanencias en el sindicato son muy amplias. Y no sólo permanencias, la gente te llama a casa. Si estuviéramos disponibles 24 horas, las 24 horas atenderíamos gente.

La importancia de esta actividad pone de manifiesto que, una vez empleados, la socialización proporciona determinados conocimientos de las reglas del entorno a los asalariados, incluidas las que tienen que ver con un tipo de defensa. También, que la explotación de los asalariados de la rama no les lleva necesariamente a la apatía y a la aceptación pasiva del orden establecido en el sector, sino a utilizar determinados procedimientos de defensa, así como ciertas prácticas de resistencia informal:

Cuando me controlas, me quieres perjudicar y discriminar, pues, respondo: «Si antes me quedaba 10 minutos, a partir de ahora, ¡no!». Hacía faenas que me pedía el cliente, pues, ahora, ¡no! Si no está limpio, limpio, pues, no pasa nada. (RASUGT)

De este modo, entre la resignación y la movilización colectiva, existen formas de defensa intermedia y de resistencia informal. En los procesos que comportan, el convenio colectivo es una referencia obligada.

\section{Todavía una eficacia convencional insuficiente, una fragmentación social}

El examen de la regulación colectiva en la limpieza de Valencia evidencia que los convenios sirven para mejorar formalmente las condiciones laborales. Ahora bien, asimismo, pone de manifiesto que la existencia de un convenio colectivo no asegura su aplicación en todas las empresas afectadas, ni el cumplimiento completo de sus cláusulas, especialmente, en las pequeñas, en las que se puede relativizar lo convenido y fijar las condiciones laborales también por la vía de la autonomía individual o de la simple arbitrariedad empresarial. A este res- 
pecto, el convenio colectivo por sí mismo no ejerce su papel de salvaguarda de las condiciones laborales de todos los asalariados de la rama. Así, se confirma el supuesto inicial sobre la insuficiente cobertura de los convenios en España, sobre todo en los centros en los que la presencia sindical y la representación de los trabajadores es débil o no existe. En este sentido, se ratifican las valoraciones efectuadas por distintos autores en España, Gran Bretaña o Francia, así como las explicaciones ofrecidas.

Y si no se aplican las normas producidas por medio de los convenios en las empresas de la rama, la homogeneización de los trabajadores sólo se consigue parcialmente, aunque se aprecia su impronta, tanto más cuanto mayor es la concentración de limpiadoras en los centros de trabajo y la presencia sindical. Por tanto, ejercen la función de integrar social y profesionalmente a los trabajadores de la limpieza de manera incompleta, con lo cual producen identidades colectivas fragmentadas. Esto no favorece la construcción de vínculos identitarios entre todos ellos.

Asimismo, se ponen de manifiesto las dificultades con las que tropiezan los trabajadores, especialmente los atípicos, para desarrollar una acción colectiva. Esta acción es más fácil a medida que los asalariados están concentrados, pero el aislamiento y la atipicidad alejan a los que padecen uno y otra del área de influencia del sindicalismo. Esta situación, que tiene raíces estructurales, intenta ser contrarrestada por los sindicalistas mediante la activación de dispositivos de permanencia jurídica para asesorar y asumir los recursos individuales que ayudan a interponer. Estos procedimientos son tanto más indispensables cuanto que constituyen, para una parte de los asalariados, el único medio a su disposición para defender sus derechos. Ahora bien, se trata de un instrumento de corte defensivo cuyo uso entraña el riesgo de convertirlo en una «arma de débiles» (Denis, 2009: 114-115), porque refleja el predominio del «hacer por los otros» sobre el «hacer conjunto». Esto se traduce en un ocultamiento de la relación colectiva. Los sindicatos padecen, pues, para superar los obstáculos de la atomización, así como para articular las distintas formas y niveles de la acción colectiva.

\section{Referencias bibliográficas}

ACITE CONSultores (2002). Estudio del sector de actividades industriales de limpieza. Madrid. Documento mimeografiado.

Alós, R.; Miguélez, F. y Recio, A. (1989a). El trabajo precario en Cataluña: La industria textil lanera del Vallés Occidental. Barcelona: CERES-CONC.

- (1989b). El trabajo precario en Cataluña: el sector del comercio. Barcelona: CERESCONC.

- (1990). Transformaciones laborales en la industria de la construcción en Catalunya. Barcelona: CERES-CONC.

Baylos, A. y Trillo, F. (coords.) (2006). Análisis de la estructura y contenidos de la negociación colectiva del sector de la limpieza de edificios y locales. Albacete: Bomarzo.

Bouffartigue, P. (1999). «Francia: ¿La norma del empleo hecha trizas? En: PRIETO, C. La crisis del empleo en Francia. Alzira (Valencia): Germania. 
DBK Consultoría (2011). Empresas de limpieza. Madrid. Documento mimeografiado.

DENIS, J.-M. (2008). "Conventions collectives: Quelle protection pour les salariés précaires?». Travail et Emploi, 116, octubre-diciembre, 45-56.

- (2009). «Dans le nettoyage, c'est le chantier qui part en grève». En: BÉROUd, S. y Bouffartigue, P. Quan le travail se précarise, quelles résistances collectives? París: La Dispute, 99-116.

ESCUDERO, R. (2004). «Reflexiones preliminares sobre la necesidad de analizar cualitativamente la negociación colectiva». En: EsCUDERO, R. (coord.). La negociación colectiva en España: una visión cualitativa. Valencia: Tirant lo Blanch, 23-51.

INE (1995). Encuesta de servicios industriales de limpieza. Madrid.

- (2008a). Directorio central de empresas. Madrid.

- (2008b). Encuesta anual de servicios. Madrid.

- (2009). Directorio central de empresas. Madrid.

- (2010). Directorio central de empresas. Madrid.

JOBERT, A. (2002). Les espaces de la négociation collective, branches et territoires. Toulouse: Octares.

Martínez LuCiO, M. (2002). «España: regular el empleo y la fragmentación social». En: Ferner, A. y Hyman, R. La transformación de las relaciones laborales en Europa. Madrid: Ministerio de Trabajo, 547-585.

Miguélez, F. (2004). «Los últimos veinte años de las relaciones laborales». Revista Mientras tanto, 93, 45-56.

Miguélez, F. y Rebollo, O. (1999). «Negociación colectiva en los noventa». En: Miguélez, F. y Prieto, C. Las relaciones de empleo en España. Madrid. Siglo XXI, 325-346.

PÉREZ INFANTE, J. I. (2003). «La estructura de la negociación colectiva y los salarios en España». Revista del Ministerio de Trabajo y Asuntos Sociales, 46, 41-97.

Reynaud, J. D. (2004). Les règles du jeu. París: Armand Colin.

SCANDELLA, F. (2009). "Renouveaux syndical dans le secteur du nettoyage: La campagne londonienne "Justice for cleaners"». En: BÉROUd, S. y BOUfFARTIGUE, P. Quan le travail se précarise, quelles résistances collectives? París: La Dispute, 117-131. 